Article

\title{
Improving Centrifugal Compressor Performance by Optimizing the Design of Impellers Using Genetic Algorithm and Computational Fluid Dynamics Methods
}

\author{
Mohammad Omidi ${ }^{1}\left(\mathbb{D}\right.$, Shu-Jie Liu ${ }^{1, *}$, Soheil Mohtaram ${ }^{2}$, Hui-Tian Lu ${ }^{3}$ and \\ Hong-Chao Zhang ${ }^{1,4}$ \\ 1 School of Mechanical Engineering, Dalian University of Technology, Dalian 116024, China; \\ insp.omidi@yahoo.com (M.O.); hong-chao.zhang@ttu.edu (Z.H.-C.) \\ 2 Institute of Soft Matter Mechanics, College of Mechanics and Materials, Hohai University, \\ Nanjing 210098, China; soheilmohtaram@gmail.com \\ 3 Department of Construction \& Operations Management, South Dakota State University, \\ Brookings, SD 57007, USA; huitian.lu@sdstate.edu \\ 4 Department of Industrial Engineering, Texas Tech University, Lubbock, TX 79409, USA \\ * Correspondence: liushujie@dlut.edu.cn; Tel.: +86-411-84709302
}

Received: 2 September 2019; Accepted: 27 September 2019; Published: 30 September 2019

check for updates

\begin{abstract}
It has always been important to study the development and improvement of the design of turbomachines, owing to the numerous uses of turbomachines and their high energy consumption. Accordingly, optimizing turbomachine performance is crucial for sustainable development. The design of impellers significantly affects the performance of centrifugal compressors. Numerous models and design methods proposed for this subject area, however, old and based on the 1D scheme. The present article developed a hybrid optimization model based on genetic algorithms (GA) and a 3D simulation of compressors to examine the certain parameters such as blade angle at leading and trailing edges and the starting point of splitter blades. New impeller design is proposed to optimize the base compressor. The contribution of this paper includes the automatic creation of generations for achieving the optimal design and designing splitter blades using a novel method. The present study concludes with presenting a new, more efficient, and stable design.
\end{abstract}

Keywords: genetic algorithm (GA); optimization; computational fluid dynamics (CFD); centrifugal compressor

\section{Introduction}

Greenhouse gases such as carbon dioxide $\left(\mathrm{CO}_{2}\right)$ are the main cause of climate change. Decreasing these emissions to prevent global warming is of crucial importance. Simultaneously the energy industry, traffic, and numerous forms of transportation all emit significant amounts of $\mathrm{CO}_{2}$. [1].

Turbomachines, including centrifugal compressors and pumps, consume a huge amount of energy in the world, both directly and indirectly [2,3]. Turbomachinery is a part of everyday life [4]. They are of central importance in various ways such as aeronautics, petrochemical processing, generation of power, refrigeration, etc. They contribute to compressors, turbochargers, pumps, turbines, and other integral equipment [4]. When applied to a wide variety of uses, even miniscule changes in efficiency and design can yield massive benefits [5]. Sustainable development will, to a certain degree, depend upon the performance improvement of turbomachinery. 
One of the most important problems with using these machines is their damping and extremely high rates of energy loss compared to other types of machines. However, due to the fluidity of the output of these machines, as well as their relatively uncomplicated design and process system, they are still far superior to other types of compressors used.

The impeller is the most important part of a turbomachine, as it plays a critical role in fluid processing, and it has therefore constantly been redesigned, optimized, and restudied over time. However, centrifugal impellers have many limitations that still need to be improved upon with redesign. Blades on the compressor impeller have always had the most significant impact on the efficiency and performance of these machine parts.

Many available resources have adopted different approaches to design impellers in centrifugal compressors [6,7]. An impeller is the rotary component of centrifugal compressors that consists of blades, which are geometrically complex due to their profile, and their flow pattern is 3D with secondary flows [8]. Given the different design parameters and flow aspects, studying impellers in centrifugal compressors is crucial. Making efforts to optimize the design of impeller as a key component is crucial given that the performance of compressors highly depends on its design [9]. Research suggests that impeller contribution to losses is significant compared to other components of a centrifugal compressor [7]. Proposing efficiency enhancement methods is therefore of great importance in this area.

The method proposed by Swine to change the impeller design [10,11], namely trimming, begins with a scratch design of impeller and proceeds to make it more delicate. This process can affect the boundaries of impellers in different directions. The direction of correction in their study was axial, radial, and stream-wise. [12] optimized compressors by redefining the curve at the hub and shroud and changing control points in the Bezier polynomial, achieving a $0.6 \%$ improvement in the pressure ratio and $1.4 \%$ improvement in efficiency. Breaking the shape and number of impellers constitutes the general secondary methods for optimizing impellers. According to this method, [13] investigated the effect of inserting two splitter blades between the main blades rather than relying on the typical setup, and could increase the mass flow rate and even efficiency and the pressure ratio. Moreover, [14] conducted an experimental study on this subject. Improvements in the performance of compressors were also reported in the literature [15], by experimentally testing impellers. [16] proposed an optimization method by changing the shape of blades, while considering the instability margin of the compressor, and found the inlet angle of the impeller to exert significant effects on the compressor efficiency. Given that the outer radius and width of the outlet contribute to instability, the shape of blades remained unchanged. [17] argued that the leading edge of sonic compressors could create shock waves when placed in an area greater than Mach one, negatively affecting splitter blades. This problem was solved by moving the starting point of the splitter forward and out of this area. [18] also proposed an optimization method for enhancing the efficiency of centrifugal compressors, which involved using the same profile for the primary and splitter blades and changing the initial profile using the Bezier polynomial points. The results suggested an efficiency increase of about 2.5\%. Moreover, [19] specified certain parameters for the objective function, including pressure ratio, efficiency and thrust, and changed the impeller profile from the hub to shroud, resulting in incredible improvements, including an efficiency increase of $4.5 \%$ and a pressure ratio increase of $16 \%$. Investigating the effect of exit pressure pulsation on the performance and stability limit of a turbocharger centrifugal compressor, [20] showed that the presence of a volume in the system exerts the most significant effect on the surge margin of the compressor under unsteady conditions. Optimizing the aerodynamic design of the impellers of a centrifugal compressor using an inverse method based on the meridional viscous flow analysis [21] resulted in performance improvements and suppression of flow separations on the suction surfaces in the optimal design cases.

The present optimization scheme specifies the changes in parameters using the computational fluid dynamics (CFD) data that correct the differences for the next steps. CFD has been widely used in the compressor designs, and there are many design considerations that need to be addressed during the 
designing process [22,23]. From a mathematical perspective, this scheme determines the direction and value of the gradient of parameters. Artificial neural network [18] and GA were used for this analysis. GA uses mechanisms such as mutation and cross-over to produce new generations of compressors [24]. This algorithm produces different versions of a compressor in different generations, and decides on terminating certain generations and refining others based on simulation results.

Due to the secondary flows in the impellers of centrifugal compressor splitter blades, the situation is not the same as that of the main blades. The flow does not match with splitter blades and this leads to performance loss in the compressors. To mitigate this loss, compressor blades should be redesigned. In this project, the changes in geometry of blade flow regimes will be analyzed. If the target is a specific compressor, this modification is limited to the impellers; otherwise, it may be necessary to change other parts, as well.

The same constraints used in previous studies with the same profile for the main and splitter blades were not used in the present research. A wider range of parameters was therefore explored when optimizing the compressor design. Two different base methods are generally used for compressor optimization. The trimming method makes small changes in the design parameters of impellers and monitors the effect of these changes on the overall performance of compressors while focusing on parameters such as Bezier points. This method is usually based on a constrained range of parameters, and is efficient in terms of computational burden, although its results are user-dependent given the need for a manual search in the computational domain of optimization.

The second method investigates major changes made in the blade shape and number and the beginning and end points of splitters. This method usually automatically examines parameters and a wide range of designs including a higher number of parameters compared to the trimming method. An optimization algorithm is integrated into this method to search in the domain of solutions and show the direction of steps towards an optimized system. In addition, given the mathematical algorithm behind optimizations, the results are unpredictable and the computational burden is heavier than that of the first method.

\section{Optimization Procedure}

Genetic algorithm is a method for optimizing a function called the objective or fitness function on a domain. The value of the objective function is calculated at randomly selected points of the domain. Each set of points form a generation, and the first generation is randomly selected from the domain. The best members of every generation with the highest objective function values can be included in the next generation. Generation members are therefore gradually improved, and this process continues until an arbitrary condition stops the algorithm.

Optimization begins with current blades of a compressor and repeatedly alters the impeller and checks the performance to achieve a better design. A code was therefore developed and an automatic process was designed. This process begins with the base compressor and is slightly modified by GA. The performance of this altered compressor is examined by simulating the flow using CFD methods, which guides GA to find the optimization direction and create a new generation. At each step, GA tests certain pre-defined geometrical design parameters of the compressor for the steps ahead. This chain of actions leads to creating different generations of the compressor. Performance is improved through generations until GA reaches the terminating point. The code helps manage this process by connecting GA to the CFD code for conducting simulations and transmitting data between the two algorithms. Although this method suits a multi-parameter system such as a compressor, flow simulations requiring many calculations make the whole process large, costly, and very time consuming in a way that the optimization approximately takes six days of the CPU time. The number of iterations is limited in CFD modelling depending on the accuracy needed and the amount of calculation allowed. However, the process termination is not enforced in GA as the algorithm automatically stops the calculations if it finds out that no further improvements in performance can be achieved. 
Figure 1 shows the efficiency optimization process of the best efficiency point (BEP) in the optimized compressor as well as some generations. The minor improvement observed in one generation is due to the similarity of all the designs belonging to that generation; nevertheless, mutation, as a GA tool, creates a new totally different generation between two generations.

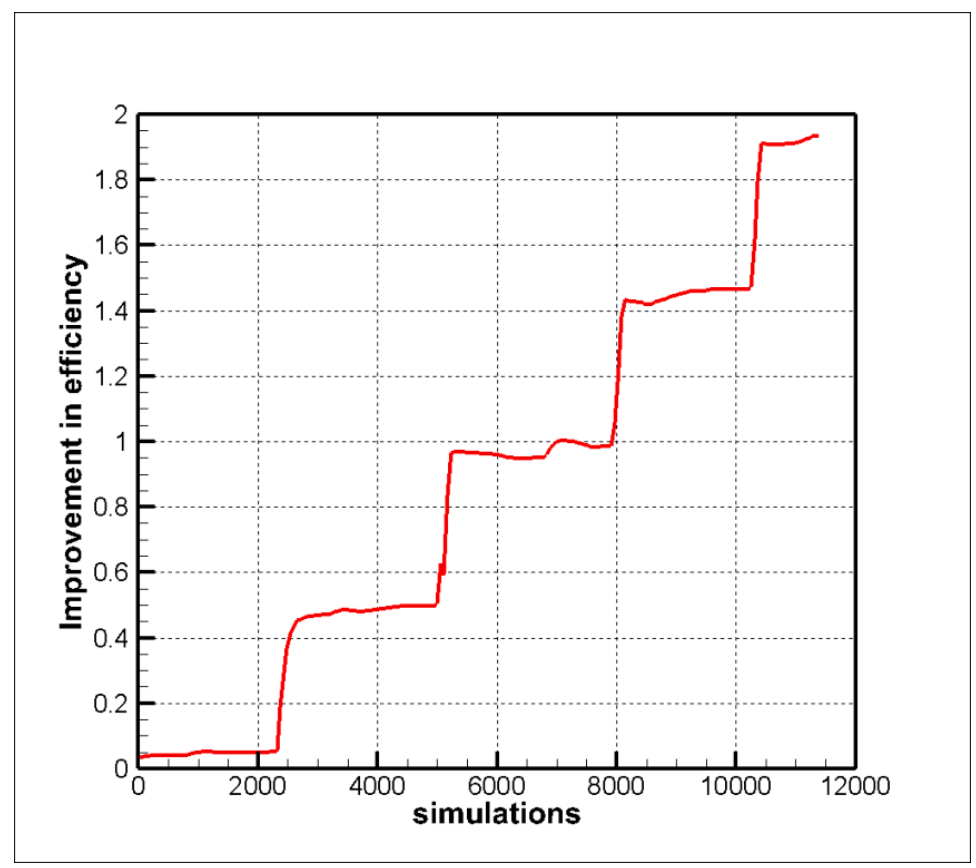

Figure 1. The efficiency (\%) optimization process.

Variations in blade profiles should be expressed as multiple interpolations, which can be modified by changing control points used by this optimization method. To create these interpolations, a 3D model of the impeller was developed in which the impeller was divided into separate parts, and each part was broken into points and estimated with an appropriate interpolation. The difficulty of grid generation is that it must suit different designs of compressors and impellers with different geometries, which need special treatment. Figure 2 shows the 3D model of the impeller.
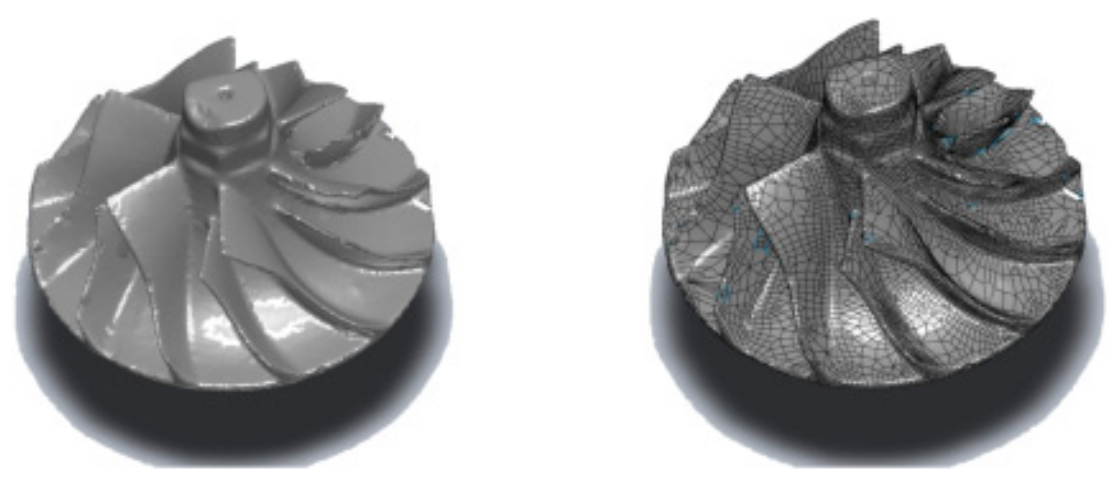

Figure 2. Three-dimensional (3D)/Meshing model of impeller.

Different designs of each compressor are analyzed by simulating the flow field through all of the machines using a commercial code. The BEP of the base compressor makes up the operating conditions for this simulation, and the comparisons are therefore valid for this point.

The design loop for the impeller forming the optimization method based on GA analyzes the parameters of flow field, including pressure ratio, efficiency and $Y+$, as the inputs into the optimization code, to create new designs [25]. 
GA seeks to minimize or maximize the objective function as a combination of performance parameters of the compressor, including pressure ratio and efficiency. The weight of each parameter in this function depends on the design objectives. Given that pressure ratio can be increased using simpler methods such as increasing the compressor diameter compared to efficiency improvement methods, maximizing efficiency is more emphasized in literature when optimizing centrifugal compressors. Only one or a number of operating points can be considered in optimizations. Although the second method cited is not very popular, it improves the performance of a compressor compared to the base design in the whole range of operation conditions rather than in a single operating point. Equation (1) shows the mass flow averaged efficiency:

$$
\phi=1-\frac{\int_{\dot{m}_{1}}^{\dot{m}_{2}} \eta d \dot{m}}{\dot{m}_{2}-\dot{m}_{1}}
$$

where $\phi$ is the objective function, $\eta$ is the efficiency and $\dot{m}$ represents the mass flow rate of the compressor and the indices show the maximum and minimum operating points of the compressor. This function indicates that the parameter that will be optimized and is a mass flow averaged efficiency in overall working points of the compressor in one rotational speed.

Variables should be determined in the optimization to specify the degree of freedom associated with the optimization parameters. The higher the number of parameters selected, the longer the calculation duration and the heavier the data processing load. Moreover, certain variables that did not significantly contribute to the results were selected at the following points for the optimization, Figure 3 shows the schematic of the following optimization variables:

- The initial point of the blades in the hub profile

- the initial point of the blades in the mid-span profile

- the initial point of the blades in the tip profile

- $\quad$ peripheral angle specifying the splitter position versus the main blades



Figure 3. Schematic of the optimization variables.

Certain constraints should be applied on these parameters to design a real optimized compressor, otherwise the optimization process will create non-physical designs. The initial point of blades is limited between the initial and ending of the main blades and the peripheral angle between two sequential blades.

Equation (2) presents the mathematical model of the problem.

$$
\text { Objective function }(\Phi)=f(a, b, c, d)
$$


Given the method selected for the optimization, a scheme is designed for achieving the goal. The optimization method involves an iterative process, and the optimization algorithm includes several steps as follows:

(1) Making curves for blades

(2) Developing 3D models of the new impeller

(3) Creating a grid for the new impeller

(4) Simulating the flow field of the impeller

(5) Analyzing the flow field and proposing new curves based on flow simulations

(6) Repeating the process

\subsection{Step 1}

For optimization of the blade, its geometry should be expressed using interpolations, which can be modified by changing control points used by the optimization method to create these interpolations. The 3D model of the impeller described earlier divides the impeller into separate parts, and each part is broken into certain points and estimated with an appropriate interpolation.

\subsection{Step 2}

The 3D model of the impeller was developed in Bladegen, which uses a file specifying all the impeller details. The optimization method alters this file to change the impeller geometry. Bladegen is a professional software used for designing turbo machines. It has special sections for radial impellers, axial turbines and compressors, IGV, vanned diffusers, etc. A design of profiles, blades, and flow channels in the compressor is developed with this software (Figure 4).

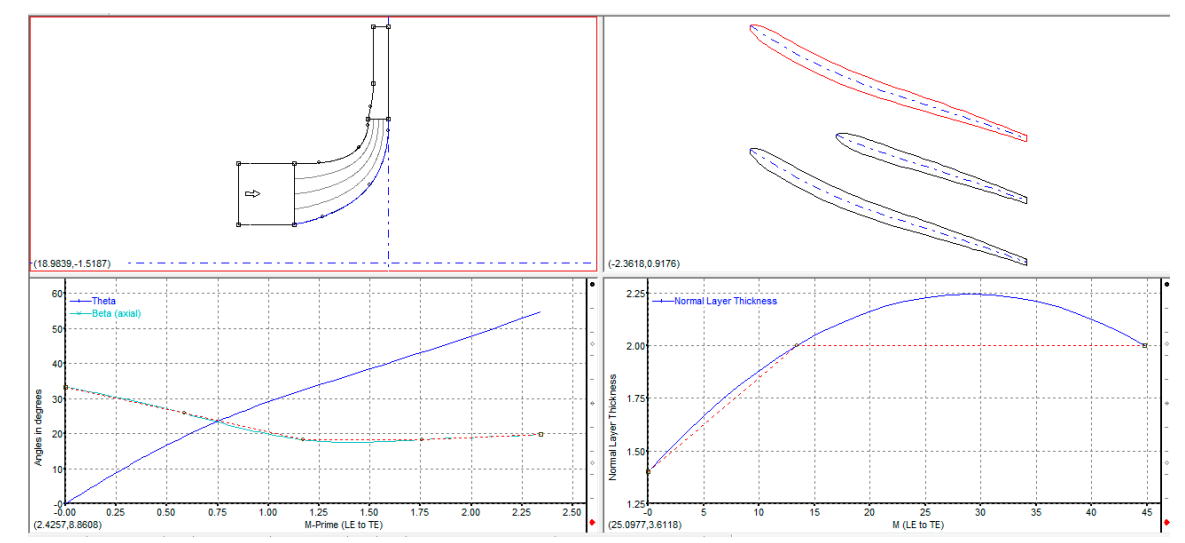

Figure 4. A design of profiles, blades, and flow channels in the compressor is developed with the Bladegen software.

\subsection{Step 3}

The grid generation method associated with compressors involves some difficulty in this step. A method and controls are required to create grids with a good quality for different compressor designs. Every geometric design of an impeller requires special treatment. Meshing procedure is one of the most important steps of 3D analysis.

A reliable meshing is sufficiently fine-grained in the areas with sharp gradients. Determining the dimensions of work is very important in turbomachines because the results will not provide converge if the meshes are too large, and the computational time will increase sharply if the meshes are too small.

Therefore, mesh size selection needs to strike a balance between the accuracy of the solution and the computational time; this step brings about a trial-and-error process. The meshing of flow domains is a tricky procedure in turbo machines because of the edges of blades and corners in channel of flow. 
Figure 5 shows the meshing of flow domain in TurboGrid. TurboGrid is especially useful for this procedure as it finds inlets and outlets of flow and, gathering information regarding the blades, builds a fine mesh proportional to each place of the domains.

The efficiency and the pressure ratio do not change significantly after a certain number of meshing, for as the computational meshes grow smaller, they suggest that the meshing computation is optimal.



Figure 5. Meshing of flow domain, TurboGrid software.

The total number of grid nodes is more than 1,250,000 that created by the structured method, and the nearest nodes to the walls are 20 micrometers far. So, the maximum $y+$ of the walls is around 30 .

\subsection{Step 4}

The compressor can be analyzed by simulating the flow field throughout the whole machine. CFX was the software used in this simulation [26]. Flow modelling is an important part of 3D simulation. The CFX is an ANSYS module for fluid simulation, yet its core is designed for turbomachines, therefore it is easier to connect the rotary parts and stationary parts of compressors than other fluid-modelling modules.

There is a BEP for the base compressor design, and optimization is usually performed at this point; nevertheless, the present research considered optimization in the whole operating range of the compressor at the nominal speed. [27-29] have shown the code validation for turbomachine flows.

The main goal of 3D modelling is to observe details of the flow. Simplifications are rare in this method, and outcomes are therefore realistic and close to actual conditions. In 3D modelling, phenomena such as backflow, vortexes, and turbulence are present that would be absent in 1D and 2D models. One of the main criteria in 3D modelling is the geometry of the flow domain.

An appropriate grid is the key to an effective and accurate modelling of flow. Grids of domain determine the cost of computation and some basic errors of modelling. The shape of grids is important especially at regions near the walls. The complex geometry of turbo machines makes grid generation all the more important. Grid generation for rotary components will be done by structured methods, and this choice renders the process more efficient except in regions such as volute, where a non-symmetric unstructured grid is suitable.

The following are important aspects which deserve attention in the simulation:

For connection of different parts, the Transient Rotor-Stator and Frozen-Rotor Simulation method will be used. This method saves details of flow and works not only on averaged flow properties. The frozen-rotor simulation is applied to attain a steady state solution for various parts of the machine. To be specific, the equipment's geometry does not change throughout the interface, and the condition is steady. 
- Flow is steady.

- The turbulence method is SST(Menter's Shear Stress Transport) [30]. This model is designed to obtain the ultimate accuracy in predicting the onset and division of mass flow under adverse pressure gradients. This model has both high performance in observing near-wall flows and secondary flows. The results of computations with this model show significant improvement in flow separation. The high performance of this model has been shown in several validation studies. SST also recommended simulating the boundary layer with high accuracy [31-33].

- The effects of turbulence were modeled using the standard $k-\varepsilon$ turbulence model. To make the simulation time economical, wall function is used to resolve the wall flows. It has merits of $k-\varepsilon$ and $k-\omega$ near and far from the walls.

Boundary conditions at the inlet are temperature and static pressure of air, and at the outlet is mass flow. At the inlet boundary, various forms of boundary condition may be applied. For instance, one may specify the mass flow rate (velocity) or total pressure. For the majority of computational cases, the mass flow rate is specified over the whole inlet face of the impeller. An approximate mass flow of $0.287 \mathrm{~kg} / \mathrm{s}$ is yielded as the average inlet velocity, while the measured state pressure is applied as an average value over the volute's outlet area. In order for the computations to assess the performance parameter effectively, total pressure is applied at the inlet. The impeller is turning with a rotational speed of $92 \mathrm{k} \mathrm{rev} / \mathrm{min}$ while the volute and diffuser are both stationary.

Following figures show some results of the CFD simulation. Figures 6 and 7 show the 3D numerical simulation results of the centrifugal compressor for the efficiency and pressure ratio. Figure 8 shows the results of CFD calculations for the compressor with streamlines.

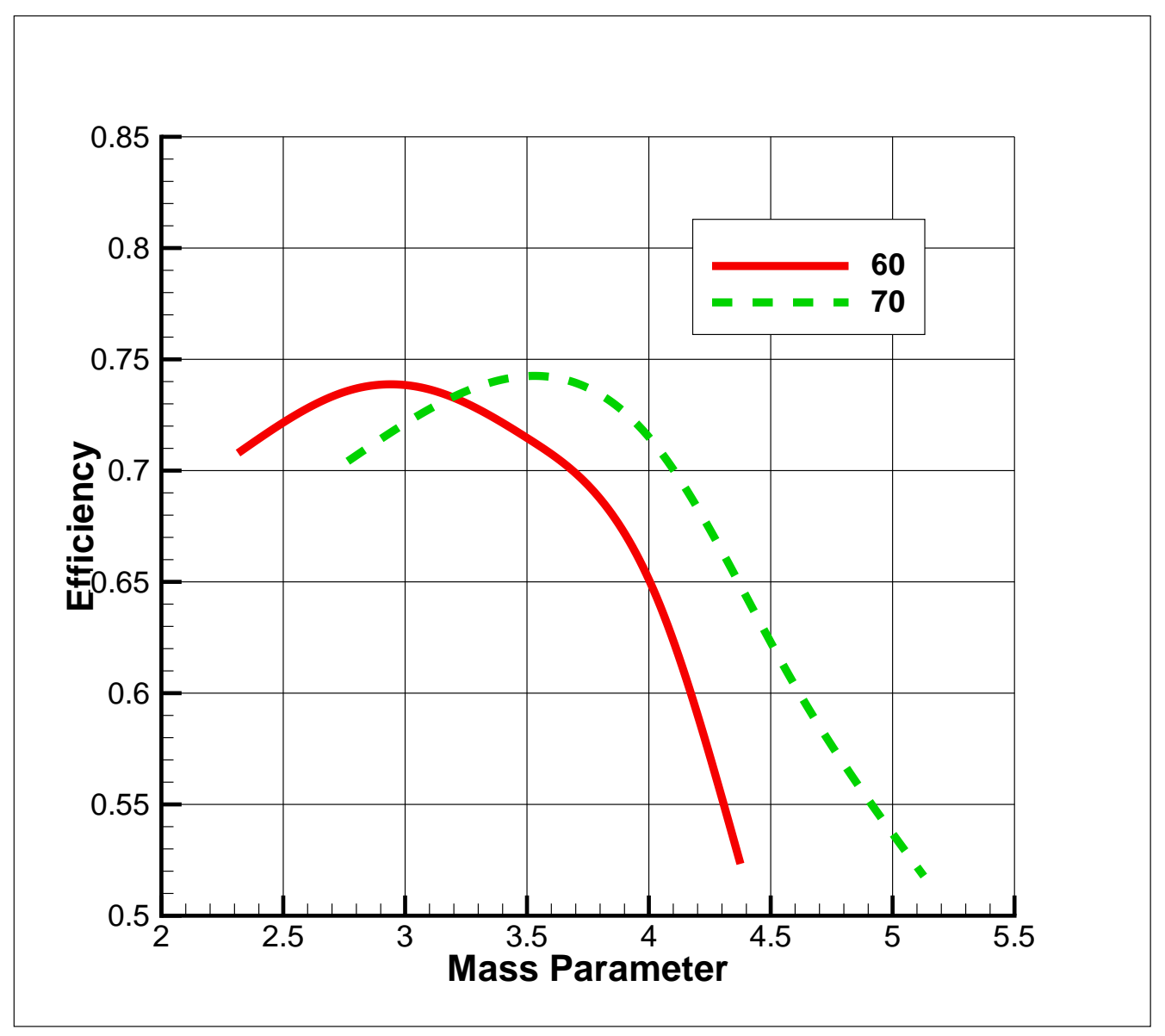

Figure 6. Efficiency (\%) by 3D simulation. 


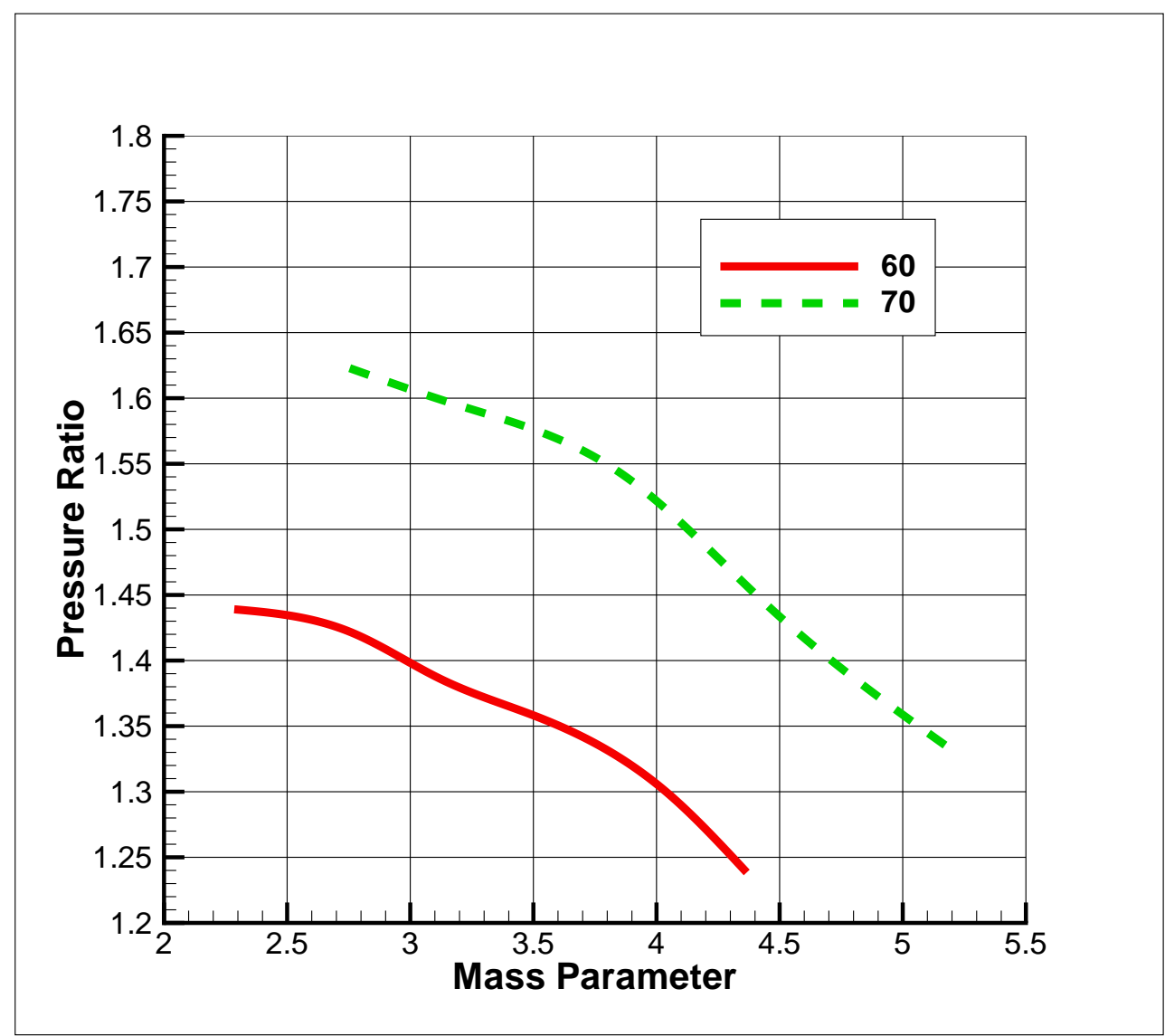

Figure 7. Pressure ratio by $3 \mathrm{D}$ simulation.



Figure 8. Streamlines in 3D simulation.

Any time one must calculate transient interaction effects at a sliding-frame change interface, a transient rotor simulation may be utilized. Such simulations are inherently always transient, as they never attain a static condition. This is because the components on each side of a transient sliding 
interface are always in relative motion with respect to each other. In a transient, sliding interface, pitch change is handled in the same way as are frozen rotor interfaces: According to the degree of pitch change across the interface, profiles in the pitch-wise direction are compressed or stretched. An increasing change of pitch will bring about rapid decreases of accuracy in computation, as is the case with the frozen-rotor condition.

\subsection{Step 5}

The design loop for impeller forming the final optimization method based on GA analyzes different impeller designs and creates new curves by investigating flow field parameters, including pressure ratio, efficiency, and $Y+$, as the inputs into the optimization method.

\section{Base Compressor}

The optimized turbocharger compressor consisted of six main blades and six splitter blades with a basic compressor geometry. In simple terms, the turbine and subsequently the compressor spins as long as the air compressor wheel spins and compressed air rotates the turbine. The BEP of this compressor is associated with a speed of $92 \mathrm{k} \mathrm{rpm}$ and an approximate mass flow of $0.287 \mathrm{~kg} / \mathrm{s}$. The nominal pressure ratio of this compressor was approximately 2.2 and its nominal efficiency was $78 \%$. Table 1 displays the compressor's specification.

Table 1. Compressor's specs.

\begin{tabular}{cc}
\hline Value & Parameters \\
\hline 2.25 & Nominal pressure ratio \\
\hline 0.28 & Nominal mass flow $(\mathrm{kg} / \mathrm{s})$ \\
\hline 92,000 & Nominal rpm \\
\hline 288 & Inlet temperature (K) \\
\hline 101 & Nominal efficiency \\
\hline $78 \%$ & Ratio of relative velocities \\
\hline 0.63 & Inlet diameter at hub (cm) \\
\hline 22 & Inlet diameter at shroud (cm) \\
\hline 54 & Number of full and splitter blades \\
\hline $6+6$ & Back swept angle (degree) \\
\hline 38 & Lean angle (degree) \\
\hline 55 & Ratio of axial length to diameter \\
\hline 0.33 &
\end{tabular}

No inlet guide vane (IGV) was present at the inlet of GT4082, although its blades were complex with splitters. The structure was therefore too complicated to be studied and improved.

\section{Validating the Results}

The accuracy and validity of the simulation method was investigated by considering the results of a reliable published article with the most details of the input parameters of the present simulation. The simulation results were compared with those obtained from the cited study. The 3D simulation of the domain was conducted without simplifying the flow. The domain simulated in the optimization process contained the inlet, the impeller, the diffuser, and the volute.

Figures 9 and 10 show this evaluation in which the results of an experimental study associated with the original geometry and a speed of $70 \mathrm{k} \mathrm{rpm}$ were used. These results were found to correctly simulate the compressor behavior and to be accurate with a maximum margin of error of $4.2 \%$ [14]. 


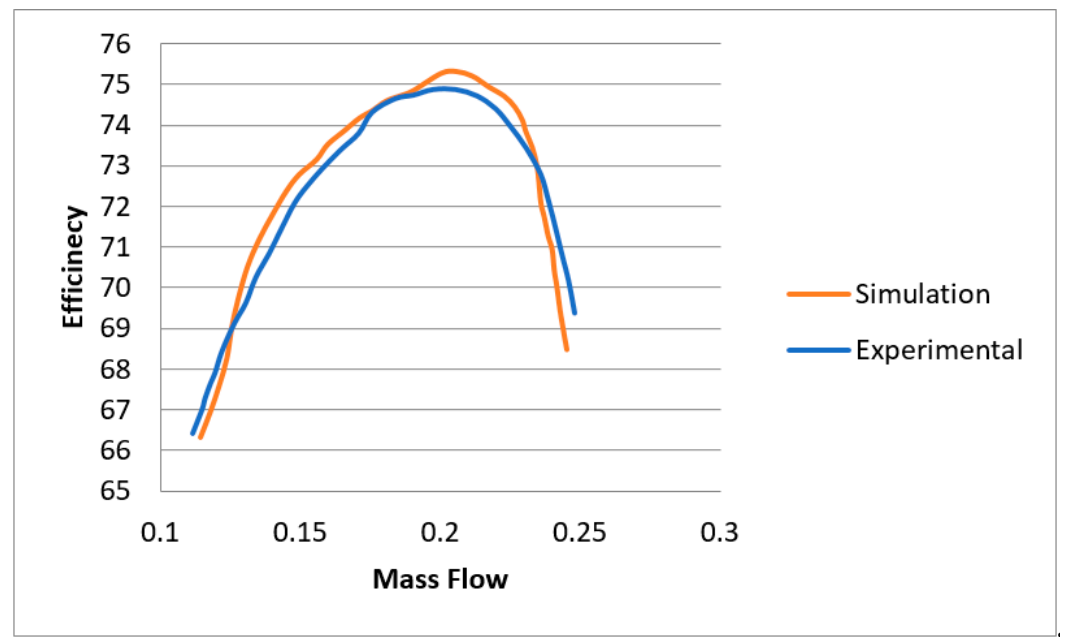

Figure 9. This is a comparison of efficiency (\%) accuracy results in the simulation with those associated with the reliable experimental study. Mass flow $(\mathrm{kg} / \mathrm{s})$.

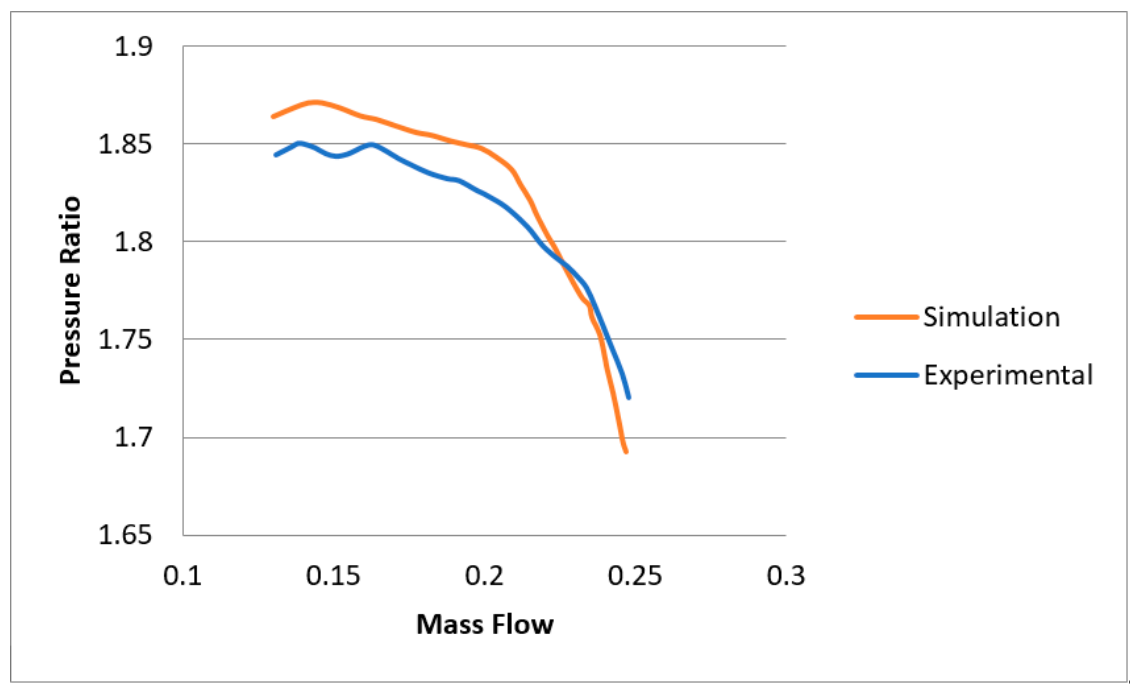

Figure 10. This is a comparison of pressure ratio accuracy results in the simulation with those associated with the reliable experimental study. Mass $\mathrm{f}$ low $(\mathrm{kg} / \mathrm{s})$.

\section{Results and Discussion}

The results obtained from running the optimization method indicate that the splitter blades should be shortened and the slope of the leading edge should be increased to improve the performance. Further investigations designed new blades which start at the middle of the hub, while their location was fixed along a $30 \%$ length of the hub in the base design. The leading edge in the optimal design has a 40-degree upward angle, while it is quite radial with a zero angle in the base design. Geometry result of the first optimization can be seen in Figure 11. 


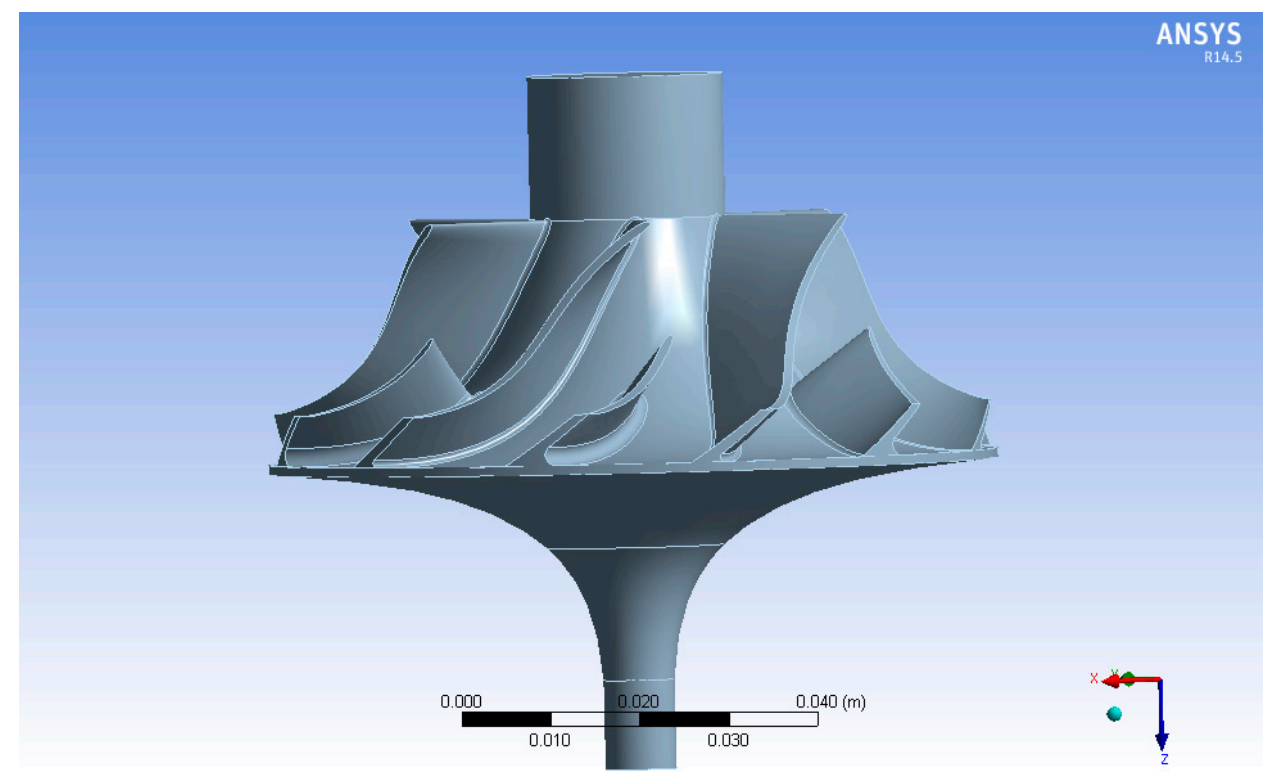

Figure 11. Geometry result of the first optimization.

Figure 12 shows variations in efficiency versus mass flow rate. The new impeller is observed to have a higher isentropic efficiency at higher mass flows. The maximum efficiency of the impeller was also found to be approximately $2 \%$ more than that of the base design.

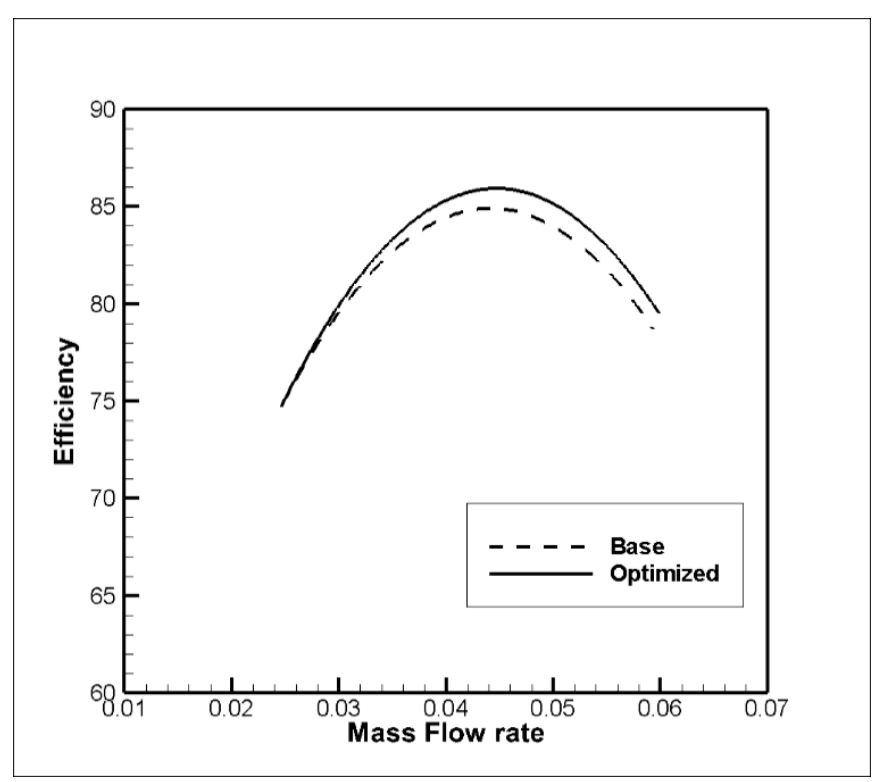

Figure 12. Comparing the efficiency (\%) of the optimized design and base design.

As may be seen from the performance curve in Figure 12, the new impeller has a higher isentropic efficiency at higher mass flows. The maximum efficiency of the impeller was also found to be approximately $2 \%$ more than that of the base design, while the pressure ratio [34], of the optimal design dropped by about $0.02 \%$, which is negligible compared to the enhancement of efficiency. The pressure ratio of the compressor can therefore be assumed to remain constant while efficiency was improved to a relatively favorable value. Figure 13 compares pressure ratios and efficiencies for the new and base designs. 


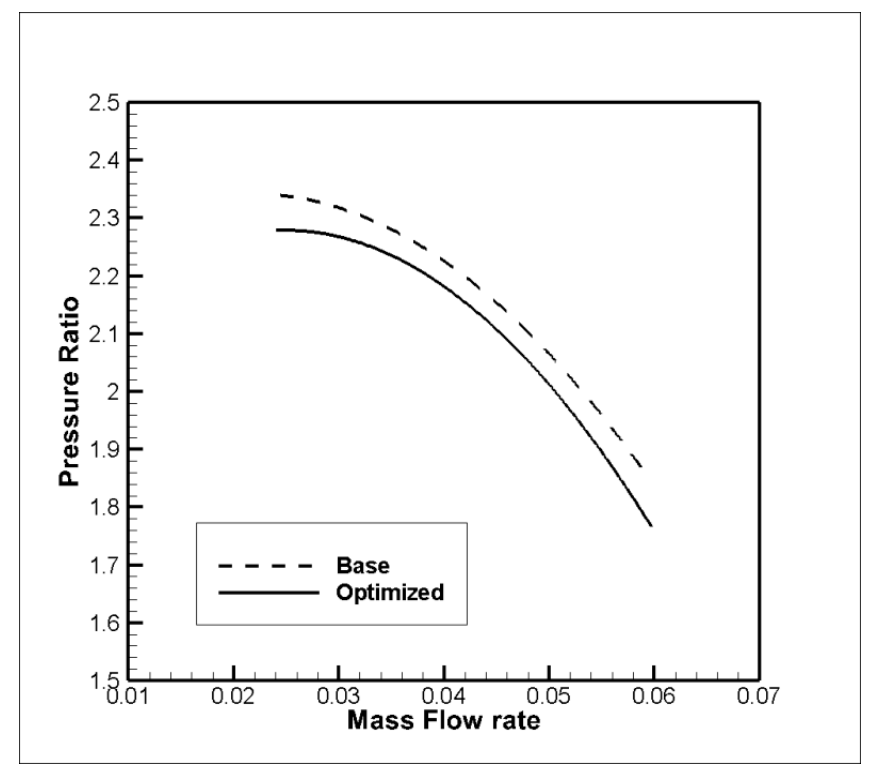

Figure 13. Comparing efficiency for the optimized design and base design.

The flow field in the hub and shroud was investigated to find the reason for the increase in efficiency. The $\mathrm{Y}+$ on the walls was not allowed to exceed 30 to maintain the numerical accuracy of the boundary layer flow in the simulation of this part.

According to Figure 14, a minor shift is observed in the hub in the beginning of the blades, followed by changes in the flow field compared to the base design. Although the flow path is smooth enough in the compressor inlet, it does not follow the blades but separates from them in the middle due to the hub profile and the Coriolis forces. At this point, splitter blades face a flow with a negative and large incident angle followed by a decrease in efficiency of the compressor, suggesting the major effect of this part on efficiency. This issue was resolved in the second impeller by shortening the blade in the optimal design and lowering the efficiency drop by preventing the flow from sharply encountering the blade.
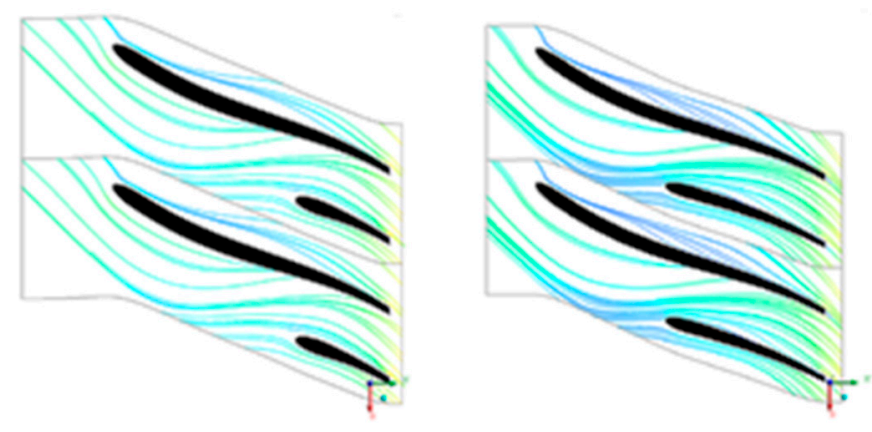

Figure 14. Comparing streamlines around impeller blades at hub. (left: Optimal; right: Base).

The shroud of the impeller can be investigated similarly. Figure 15 shows the streamlines about the shroud. Turbulence is much lower in this section and the blade positions do not cause a major drop where new blades face a smoother flow. 

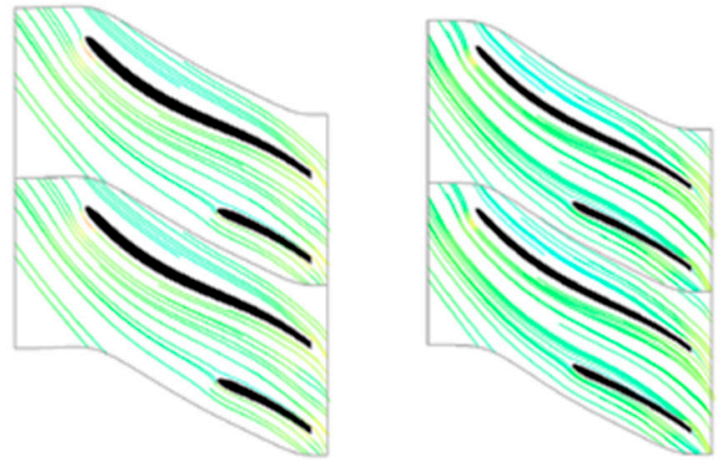

Figure 15. Comparing streamlines around impeller at shroud. (left: Optimal; right: Base).

In general, the decrease in the total length of the blades causes an efficiency improvement by reducing energy waste, while reducing the area associated with the blades and therefore reducing both the energy transfer from the blade to the flow and the pressure ratio. Simulation results suggest the simultaneous presence of both these effects, i.e., increasing efficiency is accompanied by a minor drop in the pressure ratio.

Investigation of the performance of the compressor's stall and choke is also important. Counters for the relative Mach number are shown to examine the performance of the compressor near the choking situation.

Figure 16 shows that the maximum Mach numbers emerge near the shroud section where choke initiates. In this section, a shock wave starting between two blades restricts the mass flow.
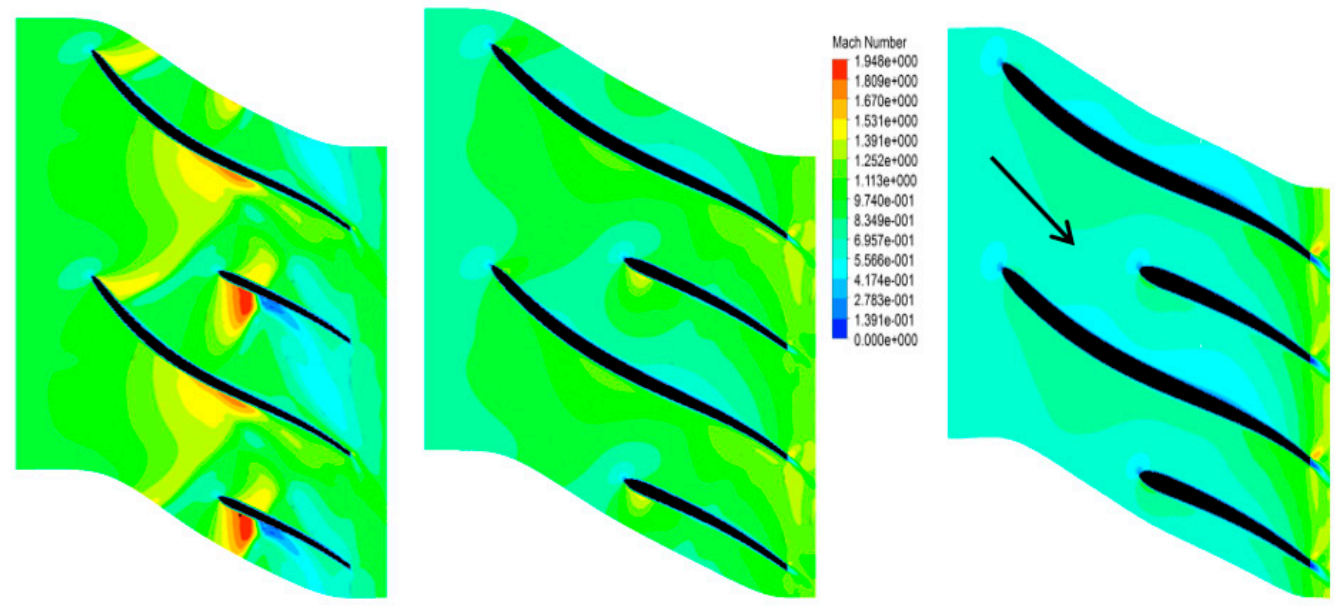

Figure 16. Mach number near the blades of the optimal impeller. (left: Shroud, center: Midspan and right: Hub).

Further studies show that this section, at the throat of the impeller, has a minimum area, and can be identified in the other conditions. The shock wave grows and entirely occupies the throat and compressor chokes with an increase in the compressor mass flow. Given that this shock wave does not emerge in the splitter area, modifying the leading edge does not make any changes in the compressor choke.

The compressor should be simulated using low mass flows to investigate the stall in the new compressor.

Figure 17 shows high turbulence and disturbed flow fields in the shrouds of both designs and inconsistent flow paths from the inlet to the outlet. Given the low mass flow and momentum, the fixed walls of the volute significantly affect the flow. The flow field shows that the mass flow cannot exit properly, which causes blockage and instability. 

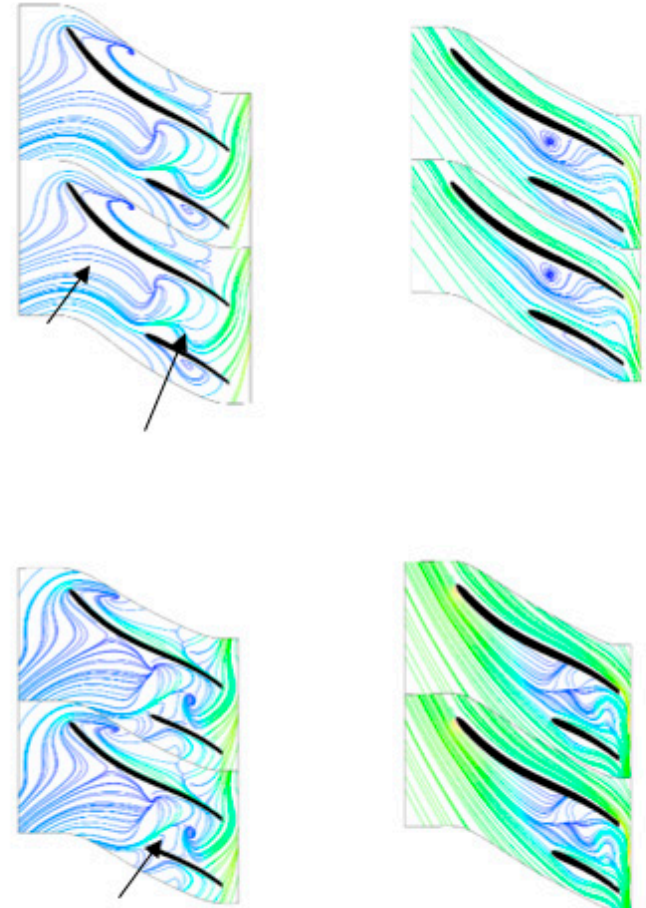
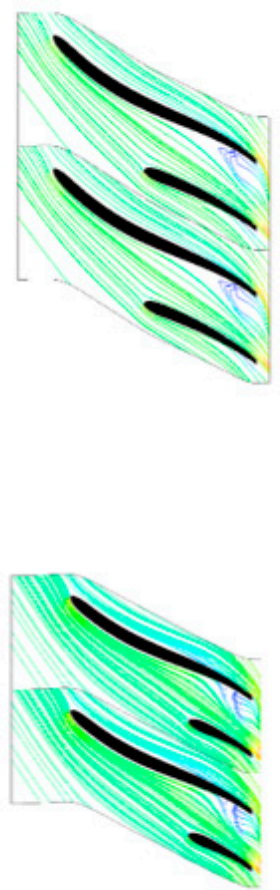

Figure 17. Streamlines around blades for optimal (up) and base design (down). (left: Shroud, center: Midspan and right: Hub).

Fewer disturbances are observed in the mid-span section and a continuous flow appears from the inlet to the outlet of the impeller. Furthermore, a large eddy flow takes a lot of space of the section, although a correct flow angle is still observed in the impeller inlet and outlet.

Major differences can be observed in the stall behavior [35] of the base and optimal designs in the hub. The most regular flow is observed in the hub section of the base design with separations, whereas in the optimal blades, the separation is postponed and the splitters present better conditions.

Tests showed that decreasing mass flow causes the mid-span and the hub quickly to become disturbed, which leads to blockage. This information can be used to create a pattern for predicting the stall and surge of compressor. Simulations suggest a 7\% postponement in the surge of mass flow in the optimal compressor.

\section{Conclusions}

The present article proposed a novel approach for optimizing the impellers of centrifugal compressors by creating and simulating different variations of the base compressor using a hybrid method comprising GA and a simulation package and defining an objective function and the variables. GA was used to develop a new compressor design by optimizing the objective function. GA, as the core of this method, was used to optimize the impeller's geometry. GA produces different generations of impellers and suggests different designs by simulating the flow using a commercial code and evaluating the performance parameters and creating generations with better performance. The optimization process began by selecting a turbocharger compressor and ended with developing a new optimal impeller design in which the pressure ratio remained constant as that of the base design and the efficiency was improved by $2 \%$, while the stability of the compressor improved against surge by approximately $7 \%$.

This improvement in efficiency also indicates that extending the study and use of CFD simulation tools as well as optimizing the performance of turbomachines can consistently be used as a reliable tool for moving forward on the path toward sustainable development. 
In the same way, future studies can construct even more conventional models by utilizing reasonable methods of optimization and continuing with more practical lab examinations. As a result of this study, it is predicted that future research will permanently increase the efficiency of the compressor by maintaining the technical specifications of the output fluid to obtain the possibility of replacing fuel-efficient engines with green and economical fuel. Through the effective combination of higher education, advanced research, and industrial practice, these findings from this paper will have a profound impact on theoretical research and commercial development in remanufacturing theory and engineering in the near future.

Author Contributions: M.O. and S.-J.L. conceived of the presented idea and drafted the manuscript. S.M. and H.-T.L. developed the theory, revised, and designed the figures H.-C.Z. and S.-J.L. supervised the findings of this work. All authors discussed the results and contributed to the final manuscript.

Acknowledgments: The authors would like to express their gratitude for the financial support provided by the National Natural Science Foundation of China (No. 51975100). This research received no external funding.

Conflicts of Interest: The authors declare no conflict of interest.

\section{References}

1. Heinrich, M.; Schwarze, R. Genetic optimization of the volute of a centrifugal compressor. In Proceedings of the 12th European Conference on Turbomachinery Fluid Dynamics and Thermodynamics, ETC 2017, Stockholm, Sweden, 3-7 April 2017.

2. Uhlmann, E.; Bilz, M.; Baumgarten, J. MRO-Challenge and chance for sustainable enterprises. Procedia CIRP 2013, 11, 239-244. [CrossRef]

3. Hall, C.A.S. The myth of sustainable development: Personal reflections on energy, its relation to neoclassical economics, and Stanley Jevons. J. Energy Resour. Technol. 2004, 126, 85-89. [CrossRef]

4. Haapala, K.R.; Zhao, F.; Camelio, J.; Sutherland, J.W.; Skerlos, S.J.; Dornfeld, D.A.; Jawahir, I.S.; Clarens, A.F.; Rickli, J.L. A Review of Engineering Research in Sustainable Manufacturing. J. Manuf. Sci. Eng. 2013, 135, 041013. [CrossRef]

5. Lindow, K.; Kaluza, A.; Stark, R. Study on sustainability developments in industrial practice. Procedia Manuf. 2018, 21, 345-352. [CrossRef]

6. Ghigliazza, F.; Casey, M.; Gersbach, F.; Robinson, C. An optimization technique for radial compressor impellers. In ASME Turbo Expo 2008: Power for Land, Sea, and Air; American Society of Mechanical Engineers: New York, NY, USA, 2008; pp. 2401-2411.

7. Japikse, D. Centrifugal Compressor Design and Performance: Course Held at Wilder, Vermont, 19.9.-23.9. 1988; Concepts Eti: Wilder, VT, USA, 1988.

8. Jop, P.; Forterre, Y.; Pouliquen, O. A constitutive law for dense granular flows. Nature 2006, 441, 727. [CrossRef] [PubMed]

9. Mohtaram, S.; Lin, J.; Chen, W.; Nikbakht, M.A. Evaluating the effect of ammonia-water dilution pressure and its density on thermodynamic performance of combined cycles by the energy-exergy analysis approach. Mechanics 2017, 23, 209-219. [CrossRef]

10. Swain, D.; Engeda, A. Performance impact of impeller blade trimming on centrifugal compressors. Proc. Inst. Mech. Eng. Part A J. Power Energy 2014, 228, 878-888. [CrossRef]

11. Swain, D.; Engeda, A. Effect of impeller blade trimming on the performance of a 5.5:1 pressure ratio centrifugal compressor. Proc. Inst. Mech. Eng. Part A J. Power Energy 2014, 228, 602-613. [CrossRef]

12. Kim, J.H.; Choi, J.H.; Husain, A.; Kim, K.Y. Multi-objective optimization of a centrifugal compressor impeller through evolutionary algorithms. Proc. Inst. Mech. Eng. Part A J. Power Energy 2010, 224, 711-721. [CrossRef]

13. Jawad, L.H.; Abdullah, S.; Zulkifli, R.; Mahmood, W. Numerical simulation of flow inside a modified turbocharger centrifugal compressor. Asian J. Appl. Sci. 2012, 5, 563-572. [CrossRef]

14. Ibaraki, S.; Matsuo, T.; Kuma, H.; Sumida, K.; Suita, T. Aerodynamics of a transonic centrifugal compressor impeller. J. Turbomach. 2003, 125, 346-351. [CrossRef]

15. Gallimore, S.J.; Bolger, J.J.; Cumpsty, N.A.; Taylor, M.J.; Wright, P.I.; Place, J.M.M. The use of sweep and dihedral in multistage axial flow compressor blading_-Part I: University research and methods development. J. Turbomach. 2002, 124, 521-532. [CrossRef] 
16. Sugimura, K.; Obayashi, S.; Jeong, S. Multi-objective optimization and design rule mining for an aerodynamically efficient and stable centrifugal impeller with a vaned diffuser. Eng. Optim. 2010, 42, 271-293. [CrossRef]

17. Lohmberg, A.; Casey, M.; Ammann, S. Transonic radial compressor inlet design. Proc. Inst. Mech. Eng. Part A J. Power Energy 2003, 217, 367-374. [CrossRef]

18. Shu, X.; Gu, C.; Xiao, J.; Gao, C. Centrifugal compressor blade optimization based on uniform design and genetic algorithms. Front. Energy Power Eng. China 2008, 2, 453. [CrossRef]

19. Guo, S.; Duan, F.; Tang, H.; Lim, S.C.; Yip, M.S. Multi-objective optimization for centrifugal compressor of mini turbojet engine. Aerosp. Sci. Technol. 2014, 39, 414-425. [CrossRef]

20. Barrera-Medrano, M.E.; Newton, P.; Martinez-Botas, R.; Rajoo, S.; Tomita, I.; Ibaraki, S. Effect of exit pressure pulsation on the performance and stability limit of a turbocharger centrifugal compressor. J. Eng. Gas Turbines Power 2017, 139, 52601. [CrossRef]

21. Oka, N.; Furukawa, M.; Yamada, K.; Itou, S.; Ibaraki, S.; Iwakiri, K.; Hayashi, Y. Optimum aerodynamic design of centrifugal compressor impeller using an inverse method based on meridional viscous flow analysis. In Proceedings of the ASME Turbo Expo 2017: Turbomachinery Technical Conference and Exposition, Charlotte, NC, USA, 26-30 June 2017.

22. Kaya, M. A CFD Based Application of Support Vector Regression to Determine the Optimum Smooth Twist for Wind Turbine Blades. Sustainability 2019, 11, 4502. [CrossRef]

23. $\mathrm{Xu}, \mathrm{C}$.; Muller, M. The development of high performance centrifugal compressor using CFD and other considerations. In Proceedings of the ASME 2017 Power Conference Joint with ICOPE-17 collocated with the ASME 2017 11th International Conference on Energy Sustainability, the ASME 2017 15th International Conference on Fuel Cell Science, Engineering and Technology, and the ASME 2017 Nuclear Forum, Charlotte, NC, USA, 26-30 June 2017.

24. Mohtaram, S.; Nikbakht, M.A. Detect tool breakage by using combination neural decision system \& Anfis tool wear predictor. Int. J. Mech. Eng. Appl. 2013, 1, 59-63.

25. Neverov, V.V.; Kozhukhov, Y.V.; Yablokov, A.M.; Lebedev, A.A. Optimization of a centrifugal compressor impeller using CFD: The choice of simulation model parameters. In IOP Conference Series: Materials Science and Engineering; IOP Publishing: Bristol, UK, 2017; Volume 232, p. 12037.

26. Elmendorf, W.; Mildner, F.; Roper, R.; Kruger, U.; Kluck, M. Three-dimensional analysis of a multistage compressor flow field. In Proceedings of the ASME 1998 International Gas Turbine and Aeroengine Congress and Exhibition, Stockholm, Sweden, 2-5 June 1998; Volume 1.

27. Eisenlohr, G.; Dalbert, P.; Krain, H.; Pröll, H.; Richter, F.-A.; Rohne, K.-H. Analysis of the transonic flow at the inlet of a high pressure ratio centrifugal impeller. In ASME 1998 International Gas Turbine and Aeroengine Congress and Exhibition; American Society of Mechanical Engineers: New York, NY, USA, 1998; p. V001T01A007.

28. Dalbert, P.; Wiss, D.H. Numerical transonic flow field predictions for NASA compressor rotor 37. In Proceedings of the ASME 1995 International Gas Turbine and Aeroengine Congress and Exposition, Houston, TX, USA, 5-8 June 1995.

29. Sekavčnik, M.; Tuma, M.; Florjančič, D. Characteristics of one stage radial centrifugal turbine. In Proceedings of the ASME 1998 International Gas Turbine and Aeroengine Congress and Exhibition, Stockholm, Sweden, 2-5 June 1998.

30. Gross-Hardt, S.; Boehning, F.; Steinseifer, U.; Schmitz-Rode, T.; Kaufmann, T.A.S. Mesh Sensitivity Analysis for Quantitative Shear Stress Assessment in Blood Pumps Using Computational Fluid Dynamics. J. Biomech. Eng. 2019, 141, 21012. [CrossRef]

31. Kang, Y.-S.; Yoo, J.-C.; Kang, S.-H. Numerical study of roughness effects on a turbine stage performance. In Proceedings of the ASME Turbo Expo 2004: Power for Land, Sea, and Air, Vienna, Austria, 14-17 June 2004; pp. 1267-1274.

32. Aligoodarz, M.R.; Karrabi, H.; Soleimani, T.M.R. Study and analysis of blade twist, lean and bow effects on the axial turbine performance. Modares Mech. Eng. 2012, 12, 9-20.

33. Menter, F.R. Two-equation eddy-viscosity turbulence models for engineering applications. AIAA J. 1994, 32, 1598-1605. [CrossRef] 
34. Mohtaram, S.; Chen, W.; Zargar, T.; Lin, J. Energy-exergy analysis of compressor pressure ratio effects on thermodynamic performance of ammonia water combined cycle. Energy Convers. Manag. 2017, 134, 77-87. [CrossRef]

35. Nikbakht, M.A.; Mohtaram, S.; Moghadas, M.H. An Investigation on the Flow Behavior in the Airfoil of a Flapping Wing. Int. J. Eng. Works 2018, 5, 138-146. 